\title{
Mathematical Basis of Sliding Mode Control of an Uninterruptible Power Supply
}

\author{
Károly Széll and Péter Korondi \\ Budapest University of Technology and Economics, Hungary \\ Bertalan Lajos u. 4-6, H-1111 Budapest, Hungary \\ szell@mogi.bme.hu; korondi@mogi.bme.hu
}

The sliding mode control of Variable Structure Systems has a unique role in control theories. First, the exact mathematical treatment represents numerous interesting challenges for the mathematicians. Secondly, in many cases it can be relatively easy to apply without a deeper understanding of its strong mathematical background and is therefore widely used in the field of power electronics. This paper is intended to constitute a bridge between the exact mathematical description and the engineering applications. The paper presents a practical application of the theory of differential equation with discontinuous right hand side proposed by Filippov for an uninterruptible power supply. Theoretical solutions, system equations, and experimental results are presented.

Keywords: sliding mode control; variable structure system; uninterruptable power supply

\section{Introduction}

Recently most of the controlled systems are driven by electricity as it is one of the cleanest and easiest (with smallest time constant) to change (controllable) energy source. The conversion of electrical energy is solved by power electronics [1]. One of the most characteristic common features of the power electronic devices is the switching mode. We can switch on and off the semiconductor elements of the power electronic devices in order to reduce losses because if the voltage or current of the switching element is nearly zero, then the loss is also near to zero. Thus, the power electronic devices belong typically to the group of variable structure systems (VSS). The variable structure systems have some interesting characteristics in control theory. A VSS might also be asymptotically stable if all the elements of the VSS are unstable itself. Another important feature that a VSS with appropriate controller - may get in a state in which the dynamics of the system can be described by a differential equation with lower degree of freedom than the original one. In this state the system is theoretically completely independent of changing certain parameters and of the effects of certain external 
disturbances (e.g. non-linear load). This state is called sliding mode and the control based on this is called sliding mode control which has a very important role in the control of power electronic devices.

The theory of variable structure system and sliding mode has been developed decades ago in the Soviet Union. The theory was mainly developed by Vadim I. Utkin [2] and David K. Young [3]. According to the theory sliding mode control should be robust, but experiments show that it has serious limitations. The main problem by applying the sliding mode is the high frequency oscillation around the sliding surface, the so-called chattering, which strongly reduces the control performance. Only few could implement in practice the robust behavior predicted by the theory. Many have concluded that the presence of chattering makes sliding mode control a good theory game, which is not applicable in practice. In the next period the researchers invested most of their energy in chattering free applications, developing numerous solutions [4-8].

According to [9]: Uninterruptible power supplies (UPS's) are being broadly adopted for the protection of sensitive loads, like PCs, air traffic control system, and life care medical equipment, etc., against line failures or other ac mains' perturbations. Ideally, an UPS should be able to deliver: 1) a sinusoidal output voltage with low total harmonic distortion during normal operation, even when feeding nonlinear loads (particularly rectifier loads). 2) The voltage dip and the recovery time due to step load change must be kept as small as possible, that is, fast dynamic response. 3) The steady-state error between the sinusoidal reference and the load regulation must be zero. To achieve these, the Proportional Integral (PI) controller is usually used [10]. However, when the system using PI controller under the case of a variable load rather than the nominal ones, cannot obtain fast and stable output voltage response. In the literature there can be found some solutions to overcome this problem [11-15].

The structure of this paper is as follows. After the introduction, the second section summarizes the mathematical foundations of sliding mode control based on the theory of the differential equations with discontinuous right-hand sides, explaining how it might be applied for a control relay. The third section shows how to apply the mathematical foundations on a practical example. The fourth section presents experimental results of an uninterruptable power supply (UPS). 


\section{Mathematical Foundations of Sliding Mode Control}

\subsection{Introductory Example}

The first example introduces a problem that can often be found in the engineering practice. Assume that there is a serial L-C circuit with ideal elements, which can be shorted, or can be connected to the battery voltage by a transistor switch (see Figure 1, where the details of the transistor switch are not shown). Assume that our reference signal has a significantly lower frequency than the switching frequency of the controller. Thus we can take the reference signal as constant.

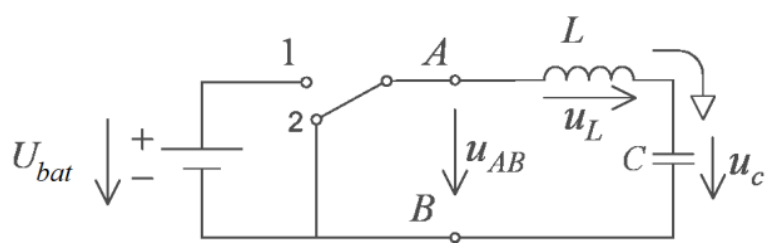

Figure 1

L-C circuit

Assume that we start from an energy free state, and our goal is to load the capacitor to the half of the battery voltage by switching the transistor. The differential equations for the circuit elements are:

$C \frac{d}{d t} u_{c}=i_{c}$ and $L \frac{d}{d t} i_{L}=u_{L}$

Due to the serial connection $i_{c}=i_{L}$, thus the differential equation describing the system is:

$u_{A B}=u_{c}+L C \frac{d^{2}}{d t^{2}} u_{c}$

Introduce relative units such way, that $L C=1$ and $U_{b a t}=1$. Introduce the error signal voltage $u_{e}=U_{r}-u_{c}$, where $U_{r}=1 / 2$ is the reference voltage of the capacitor. Thus, the differential equation of the error signal has the form:

$u=u_{e}+\frac{d^{2}}{d t^{2}} u_{e}$

,where

$u=\left\{\begin{aligned} \frac{1}{2}, & \text { if the switch is in state } 1 \\ -\frac{1}{2}, & \text { if the switch is in state } 2\end{aligned}\right.$ 
It is easy to see that the state belonging to the solution of equation (3) moves always clockwise along a circle on the phase plane $u_{e}, \frac{d}{d t} u_{e}$ (see Figure 2).

The center of the circle depends on the state of the transistor. The state-trajectory is continuous, so the radius of the circle depends on in what state the system is at the moment of the last switching. Assume that we start from the state

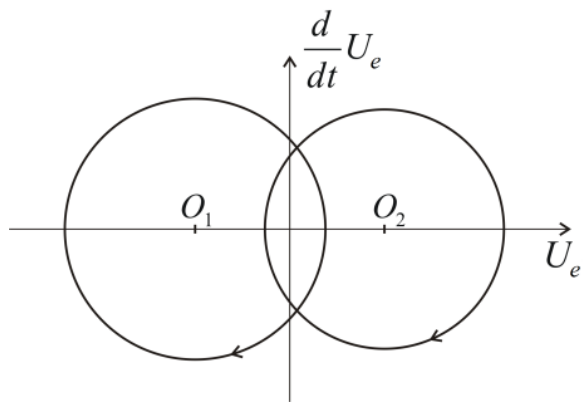

Figure 2

Possible state-trajectories

$u_{e}=1 / 2, \frac{d}{d t} u_{e}=0$ and our goal is to reach by appropriate switching the state $u_{e}=0, \frac{d}{d t} u_{e}=0$. Introduce the following switching strategy:

$u=\frac{1}{2} \operatorname{sign}(s)$

,where

$s=u_{e}+\lambda \frac{d}{d t} u_{e}$

This means that if the state-trajectory is over the $s=0$ line, then we have to switch the circle centered at $O_{1}$, if it is below the line, then we have to switch the circle centered at $\mathrm{O}_{2}$. Examine how we can remove the error. Consider Figure 3, according to (4) at first we start over the $s=0$ line on a circle centered at $O_{l}$. 


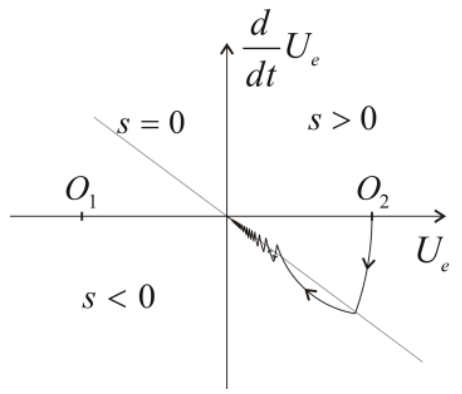

Figure 3

Removing the error

Reaching the line we switch to the circle centered at $\mathrm{O}_{2}$ so that the state-trajectory remains continuous. After the second switching we experience an interesting phenomenon. As the state trajectory starts along the circle centered at $O_{l}$, it returns immediately into the area, where the circle centered at the $\mathrm{O}_{2}$ has to be switched, but the state-trajectory cannot stay on this circle either, new switching is needed. For the sake of representation, the state-trajectory on Figure 3 reaches significantly over to the areas on both sides of the $s=0$ line. In ideal case the state trajectory follows the $s=0$ line on a curve broken in each points consisting of infinitely short sections switched by infinitely high frequency. In other words, the trajectory of the error signal slides along the $s=0$ line and therefore is called sliding mode.

Based on the engineering and geometric approach we feel that after the second switching, the behavior of the error signal can be described by the following differential equation instead of the second order (3):

$0=u_{e}+\lambda \frac{d}{d t} u_{e}$

This is particularly interesting because (5) does not include any parameter of the original system, but the $\lambda$ we have given. Thus we got a robust control that by certain conditions is insensitive to certain disturbances and parameter changes. Without attempting to be comprehensive investigate the possible effects of changing some attributes and parameters of the system. If we substitute the ideal lossless elements by real lossy elements, then the state-trajectory instead of a circle moves along a spiral with decreasing radius. If the battery voltage fluctuates, the center of the circle wanders. Both changes affect the section before the sliding mode and modifies the sustainability conditions of the sliding mode, but in both cases, the sliding mode may persist (the state trajectory cannot leave the switch line), and if it persists, then these changes will not affect the behaviour of the sliding mode of the system.

The next section will discuss how we can prove our conjecture mathematically. 


\subsection{Solution of Differential Equations with Discontinuous Right-Hand Sides}

Consider the following autonomous differential equation system:

$$
\frac{d}{d t} \mathbf{x}(t)=\mathbf{f}(\mathbf{x}(t)) \text { and } \mathbf{x}\left(t=T_{0}\right)=\mathbf{X}_{0}
$$

,where

$\mathbf{x} \in \mathfrak{R}^{n}$

$\mathbf{f}(\mathbf{x}): \mathfrak{R}^{n} \rightarrow \mathfrak{R}^{n}$

If $\mathbf{f}(\mathbf{x})$ is continuous then we can write (6) as the integral equation:

$$
\mathbf{x}(t)=\mathbf{X}_{0}+\int_{T_{0}}^{t} \mathbf{f}(\mathbf{x}(\tau)) d \tau
$$

The approach of (6) according to (7) is called Carathéodory solution, which under certain conditions may also exist when $\mathbf{f}(\mathbf{x})$ is discontinuous [16]. Recently, several articles and $\mathrm{PhD}$ theses dealt with it how to ease the preconditions which guarantee the existence of (7) concerning $\mathbf{f}(\mathbf{x})$, but for the introduced example none of the cases might be applied, we need a completely different solution.

Filippov recommends a solution, which is perhaps closer to the engineering approach described in the previous section [17], [18]. Filippov is searching the solution of (6) at a given point based on how the derivative behaves in the neighborhood of the given point, allowing even that the behavior of the derivative may completely differ from its neighborhood on a zero set, and regarding the solution ignores the derivative on this set. Filippov's original definition concerns non-autonomous differential equations, but this article deals only with autonomous differential equations.

Consider (6) and assume that $\mathbf{f}(\mathbf{x})$ is defined almost everywhere on an open subset of $\mathfrak{R}^{n}$. Assume also that $\mathbf{f}(\mathbf{x})$ is measurable, locally bounded and discontinuous. Define the set $K(\mathbf{x})$ for $\mathbf{x} \subset \mathbf{f}(\mathbf{x})$ by:

$$
K(\mathbf{x}):=\bigcap_{\delta>0} \bigcap_{\mu N=0} \operatorname{conv} \mathbf{f}(Q(\mathbf{x}, \delta)-N)
$$

,where $Q(\mathbf{x}, \delta)$ denotes the open hull with center $\mathbf{x}$ and the radius $\delta, \mu$ is the Lebesgue measure, $N$ is the Lebesgue null set and the word "conv" denotes the convex closure of the given set.

Filippov introduced the following definition to solve the discontinuous differential equation systems:

An absolutely continuous vector-valued function $\mathbf{x}(t):\left[T_{0}, T_{2}\right] \rightarrow G \in \mathfrak{R}^{n}$ is the solution of (6) if 


$$
\frac{d}{d t} \mathbf{x} \subset K(\mathbf{x}(t))
$$

for almost every $t \subset\left[T_{0}, T_{2}\right]$. Note that if $\mathbf{f}(\mathbf{x})$ is continuous, then set $K(\mathbf{x})$ has a single element for every $\mathbf{x}$, namely $\mathbf{f}(\mathbf{x})$, thus the definition of Filippov is consistent with the usual differential equations (with continuous right-hand side). However, if $\mathbf{f}(\mathbf{x})$ is not continuous, then this definition allows us searching a solution for (6) in such a domain of $\mathbf{x}$, where $\mathbf{f}(\mathbf{x})$ is not defined.

\subsection{Control Relays}

Apply the definition of Filippov as a generalization of the introductory example in the case of such a controller with state feedback, where in the feedback loop only a relay can be found (see Figure 4). Assume that the state of the system can be described by the differential equation (6) where the vectorfunction $\mathbf{f}(\mathbf{x})$ is sbtanding on the right-hand side rapidly varies depending on the state of the relay. The control (switching) strategy should be the following. In the domain of the space $G \in \mathfrak{R}^{n}$ defined by the fedback state variables define an $n$ - 1 dimensional smooth regular hypersurface $S$ (which can also be called as switching surface) using continuous $s(\mathbf{x}): \mathfrak{R}^{n} \rightarrow \Re$ scalar-vector function in the following way:

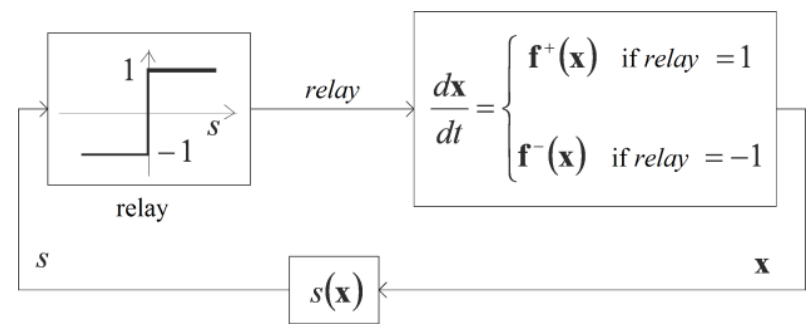

Figure 4

Controller with relay

$S:=\{\mathbf{x}: s(\mathbf{x})=0\}$

The goal of the controller is to force the state-trajectory to this surface. Mark the points of the surface $S$ with $\mathbf{x}_{\mathrm{s}}$. With the help of this surface we can divide the domain $G$ into two parts:

$$
\begin{aligned}
G^{+} & :=\{\mathbf{x}: s(\mathbf{x})>0\} \\
G^{-} & :=\{\mathbf{x}: s(\mathbf{x})<0\}
\end{aligned}
$$


Let the differential equation for $\mathbf{x}$ on domain $G$ and our switching strategy have the following form:

$$
\frac{d}{d t} \mathbf{x}=\mathbf{f}(\mathbf{x})=\left\{\begin{array}{lll}
\mathbf{f}^{+}(\mathbf{x}), & \text { if } & \mathbf{x} \in G^{+} \\
\mathbf{f}^{-}(\mathbf{x}), & \text { if } & \mathbf{x} \in G^{-}
\end{array}\right.
$$

, where $\mathbf{f}^{+}(\mathbf{x})$ and $\mathbf{f}^{-}(\mathbf{x})$ are uniformly continuous vector-vector functions.

Note that $\mathbf{f}(\mathbf{x})$ is not defined on the surface $S$, and we did not specify that $\mathbf{f}^{+}(\mathbf{x})$ and $\mathbf{f}^{-}(\mathbf{x})$ must be equal on both sides of the surface $S$.

Outside the surface $S$ we have to deal with an ordinary differential equation. Solution of (12) might be a problem in the $\mathbf{x}_{\mathbf{s}}(\mathbf{t})$ points of the surface $S$. According to definition (9), $\mathrm{K}$ is the smallest closed convex set, which you get in the following way: let's take an arbitrary $\delta>0$ hull of all $\mathbf{x}_{\mathbf{s}}(\mathbf{t})$ points of the $\mathbf{x}_{\mathbf{s}}$ belonging to the surface $S$, exclude $\mathbf{f}\left(\mathbf{x}_{\mathbf{s}}\right)$, where $\mathbf{f}(\mathbf{x})$ is not defined (remark: it is a null set $N$ domain), and we complete the set of $\mathbf{f}(\mathbf{x})$ vectors belonging to the resulting set to a closed convex set. Obviously, the smaller the value of $\delta>0$ is, the smaller the resulting closed convex set is. Finally, we need to take the intersection of the closed convex sets in the hull of all $\delta>0$ and $N$. Since $\mathbf{f}(\mathbf{x})$ is absolutely continuous, the following limits exist at any point of the surface $S$ :

$$
\begin{array}{lll}
\lim _{\mathbf{x} \rightarrow \mathbf{x}_{\mathbf{s}}} \mathbf{f}(\mathbf{x})=\mathbf{f}^{+}\left(\mathbf{x}_{S}\right) & \text { if } & \mathbf{x} \in G^{+} \\
\lim _{\mathbf{x} \rightarrow \mathbf{x}_{\mathbf{s}}} \mathbf{f}(\mathbf{x})=\mathbf{f}^{-}\left(\mathbf{x}_{S}\right) & \text { if } & \mathbf{x} \in G^{-}
\end{array}
$$

It means that the $\bigcap_{\delta>0} \bigcap_{\mu N=0} \mathbf{f}(Q(\mathbf{x}, \delta)-N)$ set belonging to any point $\mathbf{x}_{\mathbf{s}}(\mathbf{t})$ of the surface $S$ has only two elements, $\mathbf{f}^{+}\left(\mathbf{x}_{\mathbf{s}}\right)$ and $\mathbf{f}^{-}\left(\mathbf{x}_{\mathbf{s}}\right)$. We have to take the convex closure of these two vectors, which will be the smallest subset belonging to all $\delta>0$ values. In summary, the differential equation (6) with a (12) form discontinuity in the $\mathbf{x}_{\mathbf{s}}(\mathbf{t})$ points of $S$ surface according to definition (9) can be described in the following form:

$$
\frac{d}{d t} \mathbf{x}_{S}=\zeta \mathbf{f}^{+}\left(\mathbf{x}_{S}\right)+(1-\zeta) \mathbf{f}^{-}\left(\mathbf{x}_{S}\right)
$$

To illustrate (14) see Figure 5, where we drew $\mathbf{f}^{+}\left(\mathbf{x}_{\mathbf{S P}}\right)$ and $\mathbf{f}^{-}\left(\mathbf{x}_{\mathbf{S P}}\right)$ vectors belonging to the point $P$ of surface $S$. We marked the normal vector belonging to the point $P$ of the surface with $n_{p}$. The change of the state trajectory in point $\mathbf{x}_{\mathbf{S P}}$ is given by the equivalent vector $\mathbf{f}_{\mathbf{e q}}\left(\mathbf{x}_{\mathbf{S P}}\right)$, which is the convex sum of vectors $\mathbf{f}^{+}\left(\mathbf{x}_{\mathbf{S P}}\right)$ and $\mathbf{f}^{-}\left(\mathbf{x}_{\text {SP }}\right)$. 


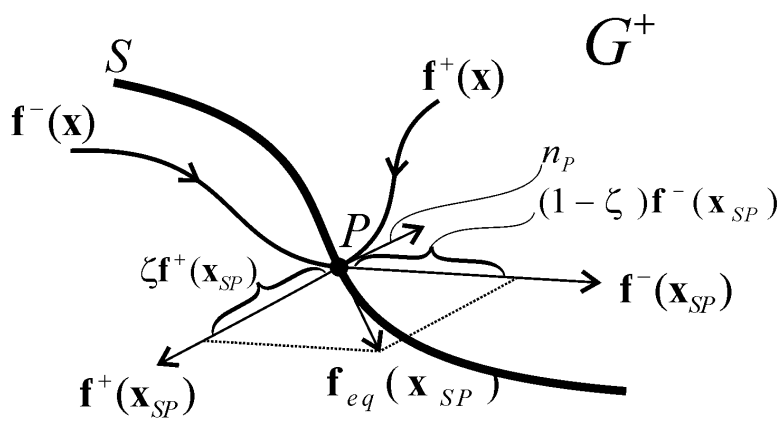

Figure 5

The state trajectory sliding along the surface $S$

Denote by $L_{f} s(\mathbf{x})$ the directional derivative of the scalar function $s(\mathbf{x})$ concerning the vector space $\mathbf{f}(\mathbf{x})$ :

$L_{f} s(\mathbf{x})=(\mathbf{f}(\mathbf{x}) \bullet \operatorname{grad}(s(\mathbf{x})))$

, where (a $\bullet$ b) denotes the scalar product of vectors $\mathbf{a}$ and $\mathbf{b}$. Since $s(\mathbf{x})$ is uniformly continuous, the following limits exist at any point of the surface $S$ :

$\lim _{\mathbf{x} \rightarrow \mathbf{x}_{\mathrm{s}}} L_{f} s(\mathbf{x})=L_{f^{+}} s\left(\mathbf{x}_{S}\right) \quad$ if $\quad \mathbf{x} \in G^{+}$

$\lim _{\mathbf{x} \rightarrow \mathbf{x}_{\mathrm{s}}} L_{f} s(\mathbf{x})=L_{f^{-}} s\left(\mathbf{x}_{S}\right) \quad$ if $\quad \mathbf{x} \in G^{-}$

The value of $\zeta$ should be defined such that $\frac{d}{d t} \mathbf{x}_{S}$ and $\mathbf{f}_{\mathrm{eq}}\left(\mathbf{x}_{\mathrm{S}}\right)$ are orthogonal to the normal vector of the surface $S$ (see Filippov's 3. Lemma [18]):

$L_{f} s\left(\mathbf{x}_{S}\right)=\left(\mathbf{f}_{e q}\left(\mathbf{x}_{S}\right) \bullet \operatorname{grad}\left(s\left(\mathbf{x}_{S}\right)\right)\right)=0$

The equation (17) can be interpreted in the following way: in sliding mode, in the $\mathbf{x}_{\mathbf{s}}$ points of the sliding surface the change of the state trajectory can be described by an equivalent $\mathbf{f}_{\mathrm{eq}}\left(\mathbf{x}_{\mathbf{S}}\right)$ vector function that satisfies condition (17). Based on (14) and (17), we obtain

$\zeta L_{f^{+}} s\left(\mathbf{x}_{S}\right)+(1-\zeta) L_{f^{-}} s\left(\mathbf{x}_{S}\right)=0$

$\zeta$ can be expressed from (18):

$\zeta=\frac{L_{f^{-}} s\left(\mathbf{x}_{S}\right)}{L_{f^{-}} s\left(\mathbf{x}_{S}\right)-L_{f^{+}} s\left(\mathbf{x}_{S}\right)}$

If $L_{f^{+}} s\left(\mathbf{x}_{S}\right)<0$ and $L_{f^{-}} s\left(\mathbf{x}_{S}\right)>0$ then on both sides of the surface $S$ the vector space $\mathbf{f}(\mathbf{x})$ points towards the surface $S$ (see Figure 6 ). Therefore, if the state 
trajectory once reaches the surface $S$, it can not leave it. The state trajectory slides along the surface and therefore this state is called sliding mode.

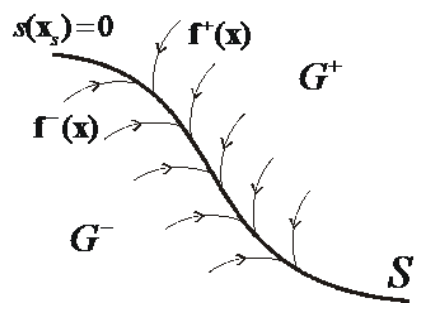

Figure 6

The $\mathrm{f}(\mathrm{x})$ vector space pointing towards the surface $S$

Note that the two conditions separately defined on both sides of the surface $S$.

$$
\begin{array}{lll}
L_{f^{+}} s(\mathbf{x})<0, & \text { if } & \mathbf{x} \in Q\left(\mathbf{x}_{S}, \delta\right) \cap G^{+} \\
L_{f^{-}} s(\mathbf{x})>0, & \text { if } & \mathbf{x} \in Q\left(\mathbf{x}_{S}, \delta\right) \cap G^{-}
\end{array}
$$

can be substituted by one inequality:

$$
\frac{d}{d t} s(\mathbf{x})^{2}<0, \quad \text { if } \quad \mathbf{x} \in Q\left(\mathbf{x}_{S}, \delta\right)-S
$$

Which can be interpreted as Lyapunov's stability criterion concerning whether the system remains on the surface $S$.

\section{The Solution of the Differential Equation of the Introductory Example}

Steps presented in Section II are applied for a circuit seen in Figure 1. As later on we can see our experimental setup for UPS (shown in Fig. 7) can be modeled with a simple L-C circuit.

There are two energy storage elements ( $\mathrm{L}$ and $\mathrm{C}$ ) in the circuit of the introductory example, therefore the behavior of the circuit can be described by two state variables. The goal is to remove the voltage error, so it is practical to choose the error signal and the first time derivative of it as the state variables.

$$
\mathbf{x}=\left[\begin{array}{c}
u_{e} \\
\frac{d}{d t} u_{e}
\end{array}\right]=\left[\begin{array}{c}
U_{r}-u_{c} \\
-\frac{d}{d t} u_{c}
\end{array}\right]
$$


The state equation of the error signal, assuming that the reference signal $U_{r}$ is constant:

$$
\frac{d}{d t}\left[\begin{array}{c}
u_{e} \\
\frac{d}{d t} u_{e}
\end{array}\right]=\left[\begin{array}{cc}
0 & 1 \\
-\frac{1}{L C} & a_{22}
\end{array}\right]\left[\begin{array}{c}
u_{e} \\
\frac{d}{d t} u_{e}
\end{array}\right]+\left[\begin{array}{c}
0 \\
1 \\
\frac{1}{L C}
\end{array}\right]\left(U_{r}-u_{A B}\right)
$$

, where $a_{22}=0$, if we neglect the losses assuming ideal $L$ - $C$ elements, while $a_{22}=$ $R / L$, if we model the losses of the circuit with serial resistance.

The design of a sliding mode controller (SMC) consists of three main steps. First, the design of the sliding surface the second step is the design of the control law which holds the system trajectory on the sliding surface and the third and key step is the chattering-free implementation [19].

Based on (4), let the scalar function defining the sliding surface be (first step in the design of the SMC):

$$
s(\mathbf{x})=\left[\begin{array}{ll}
1 & \lambda
\end{array}\right]\left[\begin{array}{c}
u_{e} \\
\frac{d}{d t} u_{e}
\end{array}\right]
$$

Rewriting the matrix equation (23) to the form (12), we obtain (second step in the design of the SMC):

$\frac{d}{d t} \mathbf{x}=\mathbf{f}(\mathbf{x})=\left\{\begin{array}{c}\mathbf{f}^{+}(\mathbf{x})=\left[\begin{array}{c}f_{1} \\ f_{2}-u_{+}\end{array}\right], \quad \text { if the switch is in state1 } \\ \mathbf{f}^{-}(\mathbf{x})=\left[\begin{array}{l}f_{1} \\ f_{2}\end{array}\right], \quad \text { if the switch is in state2 }\end{array}\right.$

,where

$f_{1}=\frac{d}{d t} u_{e}$

$f_{2}=-\frac{1}{L C} u_{e}-a_{22} \frac{d}{d t} u_{e}+\frac{1}{L C} U_{r}$

$u_{+}=\frac{1}{L C} U_{b a t}$

The directional derivative of the scalar function $\mathrm{s}(\mathrm{x})$ concerning the vector space $\mathrm{f}(\mathrm{x})$ on both sides of the surface $\mathrm{S}$ is:

$$
L_{f^{+}} s\left(\mathbf{x}_{s}\right)=\frac{d}{d t} u_{e s}+\lambda\left(-\frac{1}{L C} u_{e s}-a_{22} \frac{d}{d t} u_{e s}-u_{+}\right)=f_{1 s}+\lambda\left(f_{2 s}-u_{+}\right)
$$




$$
L_{f^{-}} s\left(\mathbf{x}_{s}\right)=\frac{d}{d t} u_{e s}+\lambda\left(-\frac{1}{L C} u_{e s}-a_{22} \frac{d}{d t} u_{e s}\right)=f_{1 s}+\lambda f_{2 s}
$$

Note that in our case $\mathbf{f}^{+}(\mathbf{x})$ and $\mathbf{f}^{-}(\mathbf{x})$ can be defined on the surface $S$, therefore we do not need to calculate the limits in (16), the points $u_{e s}, \frac{d}{d t} u_{e s}$ belonging to the surface $S$ can be directly substituted. At the same time, (21) is met only in the following domain:

$$
0<\frac{d}{d t} u_{e s}+\lambda\left(-\frac{1}{L C} u_{e s}-a_{22} \frac{d}{d t} u_{e s}\right)<u_{+}
$$

It means that, by the given control relay, only on a limited part of the surface $S$ can be in sliding mode. By completing the relay control laws with additional members, we can reach that the condition of sliding mode is fulfilled on the whole $S$ surface [20]. In case of the control relay, based on (19) and (27), we get:

$$
\zeta=\frac{f_{1 s}+\lambda f_{2 s}}{\lambda u_{+}}
$$

Based on (14), (25), (27), and (29) the differential equation describing the system in sliding mode will be:

$$
\frac{d}{d t}\left[\begin{array}{c}
u_{e s} \\
\frac{d}{d t} u_{e s}
\end{array}\right]=\frac{f_{1 s}+\lambda f_{2 s}}{\lambda u_{+}}\left[\begin{array}{c}
f_{1 s} \\
f_{2 s}-u_{+}
\end{array}\right]+\frac{\lambda u_{+}-f_{1 s}-\lambda f_{2 s}}{\lambda u_{+}}\left[\begin{array}{c}
f_{1 s} \\
f_{2 s}
\end{array}\right]=\left[\begin{array}{c}
f_{1 s} \\
-\frac{1}{\lambda} f_{1 s}
\end{array}\right]
$$

The differential equation (30) is basically the same as the equation of the sliding line, and thus we can describe the original system as a first order differential equation instead of a second order one:

$$
s\left(\mathbf{x}_{s}\right)=0=u_{e s}+\lambda \frac{d}{d t} u_{e s}
$$

This way we proved, that the state belonging to the smooth regular sliding line $S$ can be accurately followed by a state trajectory broken in each points consisting of infinitely short sections switched by infinitely high frequency. The solution of (30) is:

$$
u_{e s}=U_{e s 0} e^{-\frac{1}{\lambda} t}
$$

,where $U_{e s 0}$ is the $u_{e s}$ error signal at the moment when the state trajectory reaches the surface $S$. Based on (32) we can see that $\lambda$ is the characteristic time constant of the sliding line. Note that equation (32) does not include any parameter of the original system. This means that in the above-described ideal sliding mode the 
relay control law leads to a robust controller, insensitive to certain parameters and disturbances. The above derivation is only concerned with how the system behaves on the sliding surface, but we did not deal with the practically very important question of how to ensure that the state trajectory always reaches the sliding surface and stays on it.

Of course, in reality such an ideal sliding mode does not exist. From engineering point of view the challenge is the realization of a so-called chattering-free approximation of it.

\section{Uninterruptible Power Supply}

The measurement setup is an uninterruptable power supply. A simplified diagram of the inverter and the filter is shown in Figure 7. This circuit can be modelled as a simple L-C circuit seen in Figure 1.

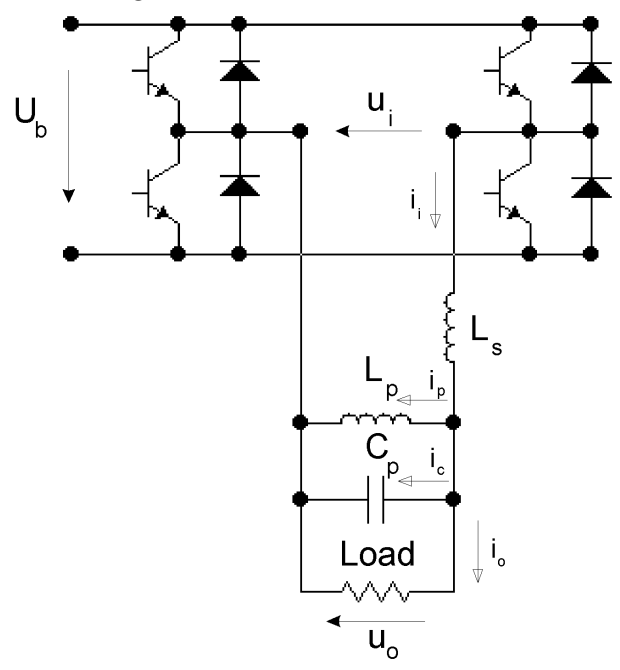

Figure 7

Simplified diagram of the inverter

Ideally the sliding mode needs an infinitely high switching frequency. The frequency is limited by a hysteresis relay. The minimum interval between two switches is determined by the hysteresis. Because of the hysteresis the error signal trajectory is chattering around the origin and harmonics will appear in the output voltage. As the third step in the design of a sliding mode controller to realize a chattering-free implementation, a proper filter is used with respect to the hysteresis, thus harmonic distortion remains at an acceptable level. Positive and negative half periods can be separated by dead-band (see Figure 8). 


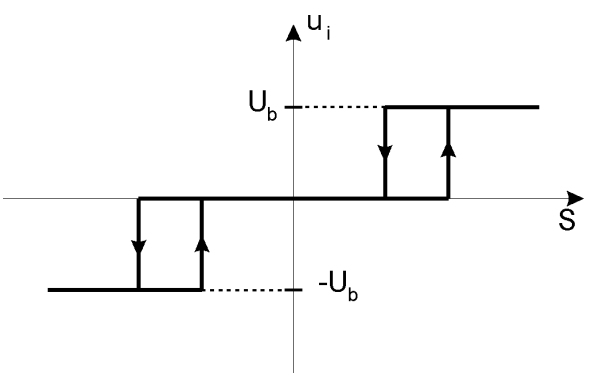

Figure 8

Dead-band hysteresis relay

As a first step the equations of the UPS shown in Figure 7 are rearranged to the form described in Section III. The load is connected to the inverter through a transformer. The $L_{s}$ in Fig. 7 is the leakage inductance of the transformer, which has a special structure to increase and set the value of $L_{s}$. The main field inductance $L p$ cannot be ignored from the point of view of the resonant circuit. Because of the main field inductance instead of (2) the filter circuit can be described by the following differential equation:

$B u_{i}=B L_{s} C_{p} \frac{d^{2} u_{o}}{d t^{2}}+u_{o}$

,where

$$
B=\frac{L_{p}}{L_{s}+L_{p}}
$$

Let us use per unit system:

$$
t_{r e l}=\frac{1}{\sqrt{B L_{s} C_{p}}} t
$$

From now on we calculate only in per unit system and do not denote it. The differential equation (33) in per unit system:

$$
B u_{i}=\ddot{u}_{o}+u_{o}
$$

, where ${ }^{-}$is the operator: $\mathrm{d} / \mathrm{dt}$. (36) corresponds to (3). Thus we can use the results described in Section III. The difference in this case is that we have more switching states and the reference signal is a sine wave. Take the influence of this sine wave into consideration.

If $u_{r e f}$ is the reference signal, we obtain the following equations:

$$
u_{\text {ref }}=U_{r e f} \sin (\omega t+\varphi)
$$




$$
\begin{aligned}
& u_{e}=u_{r e f}-u_{o} \\
& \ddot{u}_{e}=-\omega^{2} u_{r e f}-\ddot{u}_{o} \\
& \ddot{u}_{e}+u_{e}=\left(1-\omega^{2}\right) u_{r e f}-B u_{i}
\end{aligned}
$$

Solution of the differential equation:

$$
u_{e}=A_{1} \cos t+A_{2} \sin t+u_{r e f}-B u_{i}
$$

Values of constants $A_{1}$ and $A_{2}$ can be determined using the initial conditions:

$$
\begin{aligned}
& A_{1}=U_{e 0}-U_{r e f} \sin \varphi+B u_{i} \\
& A_{2}=\dot{U}_{e 0}-\omega U_{r e f} \cos \varphi
\end{aligned}
$$

, where $u_{e}(t=0)=U_{e 0}$ and $\dot{u}_{e}(t=0)=\dot{U}_{e 0}$. Using (41), the error signal trajectory can be described as follows:

$$
\left(u_{e}-u_{r e f}+B u_{i}\right)^{2}+\left(\dot{u}_{e}-\dot{u}_{r e f}\right)^{2}=A_{1}^{2}+A_{2}^{2}
$$

The curve defined by (44) can be plotted as a $\left(\mathrm{A}_{1}{ }^{2}+\mathrm{A}_{2}{ }^{2}\right)^{1 / 2}$ radius circle in the phase plane $u_{e}-\dot{u}_{e}$, the center of which runs along an ellipse given by the parameter equation system below:

$$
\begin{aligned}
& u_{e}=U_{r e f} \sin (\omega t+\varphi)-B u_{i} \\
& \dot{u}_{e}=\omega U_{r e f} \cos (\omega t+\varphi)
\end{aligned}
$$

The ellipse has three possible positions in the phase plane, depending on the value of $u_{i}$ (see Figure 9).

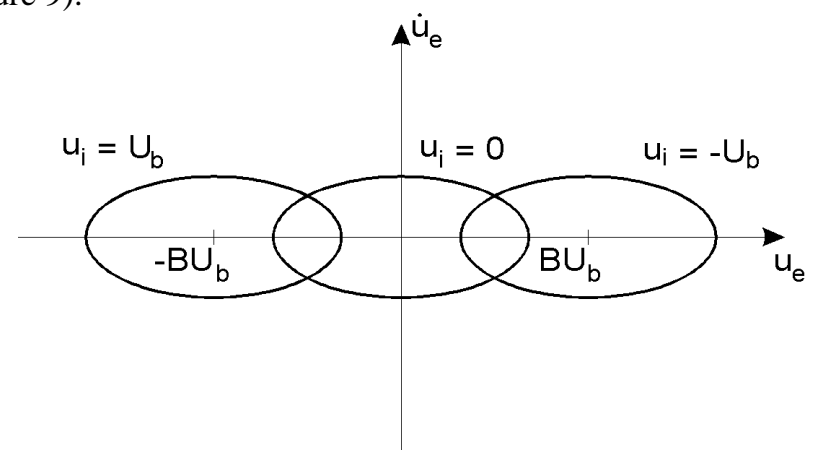

Figure 9

Ellipses describing the movement of the center of the circle 


\subsection{Robustness Analysis}

Let us check the condition of the sliding mode based on (21). Deriving (24) and eliminating the second order term using (40), we obtain:

$\dot{s}(\mathbf{x})=\dot{u}_{e}+\lambda\left[U_{r e f}\left(1-\omega^{2}\right) \sin (\omega t+\varphi)-B u_{i}-u_{e}\right]$

(47) corresponds to (5). According to Lyapunov's stability, if $s(\mathbf{x})>0$ (similar to (28)):

$\dot{u}_{e}-\lambda u_{e}<-\lambda U_{r e f}\left(1-\omega^{2}\right) \sin (\omega t+\varphi)+\lambda B u_{i}$

If $s(\mathbf{x})<0$ :

$\dot{u}_{e}-\lambda u_{e}>-\lambda U_{r e f}\left(1-\omega^{2}\right) \sin (\omega t+\varphi)+\lambda B u_{i}$

Both inequalities define a half plane, and the boundaries are perpendicular to the sliding surface changing their position according to a sine wave. The phase plane is divided into four sections (see Figure 10).

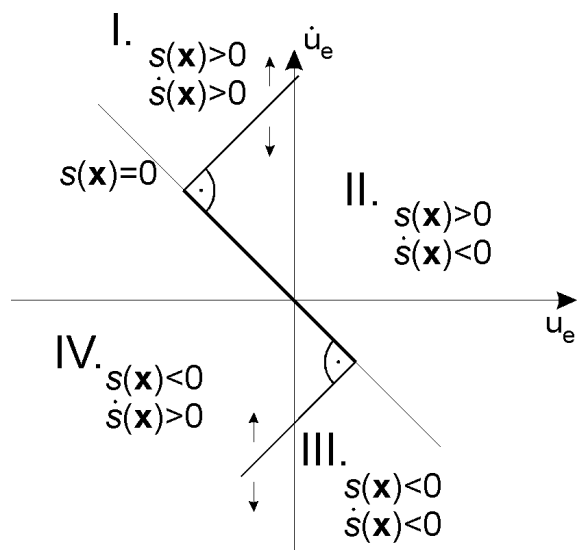

Figure 10

Phase plane of the error signal

Introduce the following switching strategy:

$u_{i}=\left\{\begin{array}{cc}U_{b}, & \text { if } s(\mathbf{x})>0 \\ -U_{b}, & \text { if } s(\mathbf{x})<0\end{array}\right.$

Usually $U_{\text {ref }}<U_{b}$ and $\omega<1$. Thus the half planes defined by (48) and (49) always have an intersection which goes through the origin where (21) is valid on both sides of the switching surface. The sliding mode occurs on the common boundary of sections II. and IV. 


\subsection{Experimental Verification}

Using the sliding mode control an experimental measurement is carried out for the UPS. The parameters of the experimental setup:

$$
\begin{array}{llll}
\mathrm{S}_{\mathrm{n}}=10 \mathrm{kVA} & \mathrm{U}_{\mathrm{n}}=230 \mathrm{~V} & \omega=314 \mathrm{rad} / \mathrm{sec} & \lambda=1 / 24 \\
\mathrm{~B}=0,9 & \mathrm{~L}_{\mathrm{s}}=0,35 \mathrm{mH} & \mathrm{C}_{\mathrm{p}}=3200 \mu \mathrm{F} & \mathrm{L}_{\mathrm{p}}=3,2 \mathrm{mH}
\end{array}
$$

Based on (32) and the per unit system (35) the characteristic time constant of the sliding line:

$$
T=\sqrt{B L_{s} C_{p}} \lambda=42 \mu \mathrm{sec}
$$

During the measurement the current and the voltage of the load are measured. Operational amplifiers are used to provide the value of $S(t)$, which signal of the controller is measured with an oscilloscope. Using the notation of Figure 7 the measurements with the oscilloscope are shown in Figure 11 and 13. The harmonic distortion is less than $1.5 \%$. The error trajectory cannot be measured directly, thus they are modeled by computer simulation. Figure 12 shows the steady-state trajectory and switching lines of the dead-band hysteresis relay. Switching delays can also been seen.

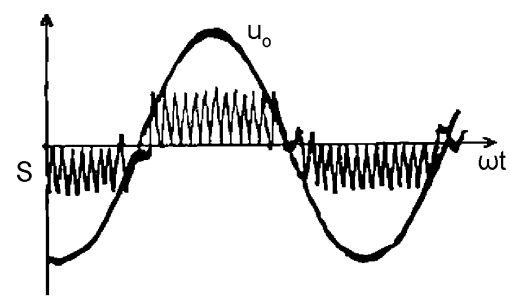

Figure 11

Experimental results for steady-state

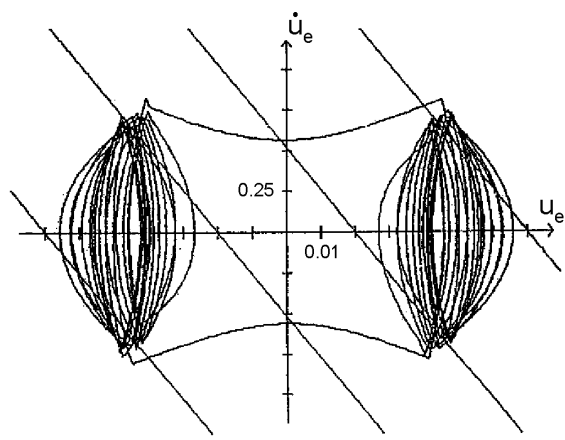

Figure 12

Steady-state error-trajectory 
Figure 13 and Figure 14 show the system's response for a $100 \%$ ohmic step change in the load. As we can see first the system has to reach the sliding surface and then it can follow the switching strategy of the case above. It takes only some switching, about $10 \%$ of the time period until the system settles.

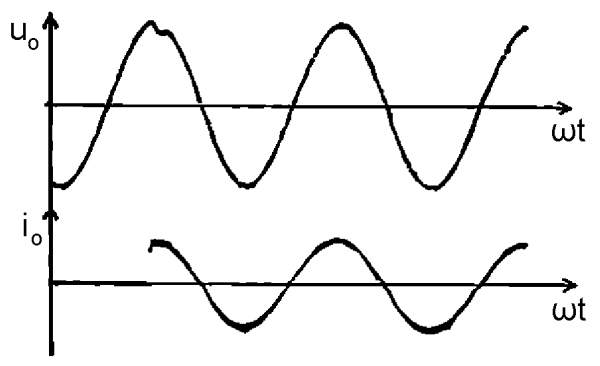

Figure 13

Experimental results for step-change

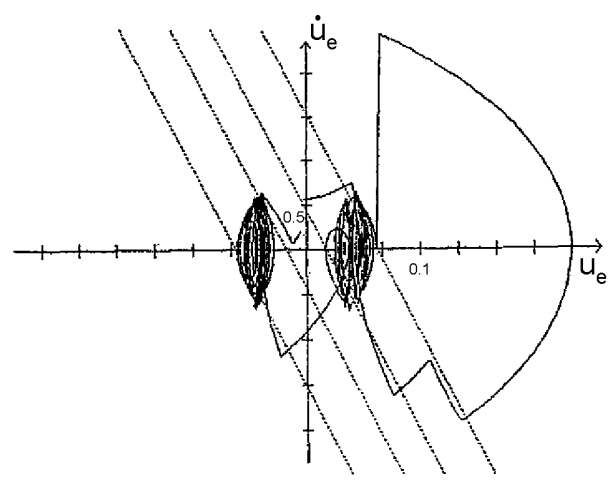

Figure 14

Step-change error-trajectory

\section{Acknowledgements}

The authors wish to thank the support to the Hungarian Automotive Technicians Education Foundation, to the Hungarian Research Fund (OTKA K100951), and the Control Research Group of HAS. The results discussed above are supported by the grant TÁMOP-4.2.2.B-10/1--2010-0009.

\section{Conclusions}

When a VSS is in sliding mode its trajectory lies on the switching line. The theoretical sliding mode is an idealization. In practice, when switching delays are present the steady state trajectory chatters around the origin. In spite of the chattering the harmonic distortion is less than $2 \%$. The response time for step change of the load is very short due to the instantaneous nature of the sliding mode control. Apart from the short transient process the uninterruptable power 
supply is insensitive to the load variation. Though sliding mode controller is very simple, it provides a good performance. By the application of the definition of Filippov to the UPS, the paper presented a practical application of the theory of differential equation with discontinuous right hand side proposed by Filippov

\section{References}

[1] P. Bauer, R. Schoevaars: Bidirectional Switch for a Solid State Tap Changer, $34^{\text {th }}$ Annual IEEE Power Electronics Specialists Conference. Vol. 1-4, 2003, pp. 466-471

[2] V. I. Utkin: Variable Structure Control Optimization, Springer-Verlag, 1992

[3] K. D. Young: Controller Design for Manipulator using Theory of Variable Structure Systems, IEEE Trans. on System, Man and Cybernetics, Vol. SMC-8, 1978, pp. 101-109

[4] H. Yu, U. Ozguner: Adaptive Seeking Sliding Mode Control, IEEE American Control Conference, Minneapolis, USA, 2006

[5] En-Chih Chang, Fu-Juay Chang, Tsorng-Juu Liang, Jiann-Fuh Chen: DSPBased Implementation of Terminal Sliding Mode Control with Grey Prediction for UPS Inverters, the $5^{\text {th }}$ IEEE Conference on Industrial Electronics and Applications (ICIEA) , 2010, pp. 1046-1051

[6] R. A. Hooshmand, M. Ataei, M. H. Rezaei: Improving the Dynamic Performance of Distribution Electronic Power Transformers Using Sliding Mode Control, Journal of Power Electronics, Vol. 12, 2012, pp. 145-156

[7] F. F. A. van der Pijl, M. Castilla, P. Bauer: Adaptive Sliding-Mode Control for a Multiple-User Inductive Power Transfer System Without Need for Communication, IEEE Transactions on Industrial Electronics, Vol. 60, 2013, pp. 271-279

[8] Yung-Tien Liu, Tien-Tsai Kung, Kuo-Ming Chang, Sheng-Yuan Chen: Observer-based Adaptive Sliding Mode Control for Pneumatic Servo System, Precision Engineering 37, 2013, pp. 522-530

[9] Tsang-Li Tai, Jian-Shiang Chen: UPS Inverter Design Using DiscreteTime Sliding-Mode Control Scheme, IEEE Trans. Ind. Electron., Vol. 49, No. 1, Feb. 2002

[10] R. Caceres, R. Rojas, O. Camacho: Robust PID Control of a Buck-Boost UPS Converter, Proc. IEEE INTELEC'02, 2000, pp. 180-185

[11] Khalifa Al-Hosani, Andrey Malinin, Vadim I. Utkin: Sliding Mode PID Control of Buck Converters, The European Control Conference, 2009

[12] Hongmei Li, Xiao Ye: Sliding-Mode PID Control of DC-DC Converter, the $5^{\text {th }}$ IEEE Conference on Industrial Electronics and Applications (ICIEA) 2010, pp. 730-734 
[13] S. Saraswathy, K. Punitha, D. Devaraj: Implementation of Current Control Techniques for Uninterruptable Power Supply, International Conference on Circuits, Power and Computing Technologies, 2013, pp. 589-595

[14] F. Tahri, A. Tahri, A. Allali, S. Flazi: The Digital Self-Tuning Control of Step a Down DC-DC Converter, Acta Polytechnica Hungarica, Vol. 9, No. 6, 2012, pp. 49-64

[15] A. Tahri, H. M. Boulouiha, A. Allali, T. Fatima: A Multi-Variable LQG Controller-based Robust Control Strategy Applied to an Advanced Static VAR Compensator, Acta Polytechnica Hungarica, Vol. 10, No. 4, 2013, pp 229-247

[16] D. C. Biles, P. A. Binding: On Caratheodory's Conditions for the Initial Value Problem, Proceedings of the American Mathematical Society, Volume 125, Number 5, May 1997, pp. 1371-1376

[17] A. G. Filippov: Application of the Theory of Differential Equations with Discontinuous Right-hand Sides to Non-linear Problems in Automatic Control, $1^{\text {st }}$ IFAC Congr. Moscow, 1960, pp. 923-925

[18] A. G. Filippov: Differential Equations with Discontinuous Right-hand Side, Ann. Math Soc. Transl., Vol. 42, 1964, pp. 199-231

[19] B. Takarics, G. Sziebig, B. Solvang, P. Korondi: Multimedia Educational Material and Remote Laboratory for Sliding Mode Control Measurements, Journal of Power Electronics, Vol. 10, 2010, pp. 635-642

[20] F. Harashima, T. Ueshiba, H. Hashimoto: Sliding Mode Control for Robotic Manipulators, $2^{\text {nd }}$ Eur. Conf. on Power Electronics, Grenoble Proc., 1987, pp. 251-256 